\title{
observaciones acterca de la \\ dosificación de cementos en morteros \\ y hormigones fraguados
}

\author{
J. CALLEJA, Or. en Ciencios Quimicas
}

\section{regsumem}

Los mélodos al uso para determinar la dosificación de conglomerantes en conglomerados, y más concretamente de cemento (portland o no) en morteros y hormigones fraguados, tienen en general limitaciones que les hacen adolecer, en la mayoría de los casos prácticos, de errores considerables.

En circunstancias determinadas generalmente imprevistas, estos errores pueden aumenlar notablemente e invalidar los resultados y las conclusiones que de los mismos pudieran extraerse.

En evitación de ello es preciso entonces complementar los métodos con otras determinaciones, particularmente si el dictamen basado en el resultado hallado para la dosificación puede afectar scriamente a intereses económicos, o determinar una responsabilidad de cualquier tipo.

\section{generalidades}

Harto conocido y frecuente es, por un motivo u otro, y de ordinario a causa de problemas legales ligados a la construcción, el caso de tener que determinar la cantidad de conglomerante con que, en su momento, fue hecho un conglomerado hidráulico dado. Es el problema de "hallar la dosificación de cemento en un mortero u hormigón fraguado".

El caso es fácil o difícil según los datos de que se disponga para su solución, y de ellos depende también la aproximación o el error de que aquélla esté afectada. Hay que decir que casi nunca las circunstancias son tan propicias como para poner a disposición del técnico encargado de resolver tales problemas, no ya todos los datos posibles, sino ni siquiera los necesarios para ello, siendo preciso, en suplencia de los mismos, establecer hipótesis más o menos de acuerdo con una realidad que siempre se desconoce. De aquí que los erro- * res puedan llegar a ser tan grandes que invaliden los resultados en algunos casos y, por lo tanto, también las consecuencias que de ellos pudieran deducirse. 
Las hipótesis mencionadas van generalmente implicitas en los métodos establecidos para hallar la dosificación, y podría decirse que implican un error sistemático mayor o menor, pero fácilmente evaluable en cuanto a su cuantía máxima. La complicación surge cuando es preciso sentar otras hipótesis complementarias, cuando se trata de "casos inter. medios" poco o mal definidos, y cuando se dan otras circunstancias con las que, por lo inesperado, no cabe contar. Entonces pueden aparecer errores "accidentales" de tal magnitud que para nada sirven los métodos ni los resultados que éstos pudieran proporcionar.

\section{fundamento de los métodos para hallar la dosifictación [1]}

El fundamento químico de los métodos para hallar la dosificación de conglomerante en un conglomerado fraguado estriba en la determinación en el conglomerado de un elemento - bien sea como tal, o bien en forma de un compuesto definido- que forme parte del conglomerante y no se encuentre en el resto de los materiales que constituyen el conglomerado, tales como los áridos y las eventuales adiciones añadidas a este último, y no presentes previamente en el conglomerante. Este es el caso ideal más sencillo, cuya solución es inmediata, si se conoce el análisis del conglomerante, o se dispone de éste para determinarlo. En tal caso la relación en peso entre el conglomerante y el conglomerado es igual a la relación entre el tanto por ciento ponderal del elemento (o compuesto) en cuestión en el conglomerante, y el tanto por ciento ponderal de dicho elemento (o compuesto) en el conglomerado. Si no se conoce el análisis del conglomerante, ni se dispone de éste para la determinación de aquél, el problema queda sin solución, a menos que, transigiendo con los errores que ello implica, se asigne al conglomerante un contenido medio, para los de su tipo y clase, del elemento (o compuesto) sobre el que se base la dosificación. El error máximo que así se puede cometer es de naturaleza sistemática y puede evaluarse con facilidad.

Un ejemplo correspondiente a este caso (1) lo constituye la determinación de la dosificación de conglomerante en conglomerados hechos con áridos calizos (calizas prácticamente puras) o con áridos exentos de sílice soluble y de cal (cantos rodados de río, sílex o pedernal). En ambos casos puede basarse la dosificación en la determinación del contenido de sílice soluble; en el segundo puede basarse igualmente en la determinación del contenido de óxido cálcico. Si se conoce por el análisis, o puede determinarse mediante él en una muestra del conglomerante el contenido de sílice soluble o de cal del mismo, en cada caso, se puede hallar la dosificación con gran precisión; en caso contrario, hay que asignar al conglomerante un contenido (medio), bien sea de sílice soluble, o bien de óxido cáleico. Supóngase que se le asigna un valor de sílice soluble de $20,5 \%$, medio aproximado entre valores extremos de 18,0 y 23,5 \% - - caso de un cemento portland (2)_; entonces el error cometido, por exceso en un caso y por defecto en otro, puede llegar a ser del orden del $12 \%$, o del $14,5 \%$, si se expresa la dosificación en kilogramos de cemento por metro cúbico de conglomerado, y se admite como densidad real de éste 2,25. En efecto, la dosificación calculada, en un caso y otro seria: 


$$
D c=1.000 \times 2,25 \times \frac{S h}{S c}=2.250 \frac{S h}{20,5}=109,75 \mathrm{Sh}
$$

siendo $D c$ la dosificación calculada $\left(\mathrm{kg} / \mathrm{m}^{3}\right.$ y $S h$ y $S c$ la sílice soluble del conglomerado y del conglomerante respectivamente; la dosificación real sería:

$$
D r_{1}=1.000 \times 2,25 \times \frac{S h}{S c}=2.250 \frac{S h}{18}=125,00 S h
$$

en un caso, $\mathrm{y}$ :

$$
D r_{2}=1.000 \times 2,25 \times \frac{S h}{S c}=2.250 \frac{S h}{23,5}=95,75 S h
$$

en el otro. Las diferencias $D c-D r$ serían:

$$
I) c-D r_{1}=109,75 S h-125,00 S h=-15,25 . S h
$$

y

$$
D c-D r_{2}=109,75 S h-95,75 S h=+14,50 S h
$$

y los errores relativos en tanto por ciento serían:

$$
\frac{-15,25 S h}{125,00 S h} \times 100=-12 \%
$$

y

$$
\frac{+14,00 S h}{95,75 S h} \times 100=+14,5 \%
$$

Análogas consideraciones pueden hacerse si la dosificación se basa en el contenido (determinado, conocido o supuesto) de óxido cálcico en el conglomerante, y determinado en el conglomerado.

A los principios expuestos responde la determinación de la dosificación según la correspondiente norma de la ASTM (3), y según la NELC 5.01-a española (4), que es una transcripción de la anterior. Esta última admite como valor medio de la sílice soluble para los cementos españoles, cuando no se conoce ni hay medio de determinarla, 20,5 \%. Si se conoce o se puede determinar la sílice soluble del conglomerante, el método puede aplicarse cualquiera que sea éste, y el error que afecta a la dosificación hallada puede ser del $4 \%$; si no se conoce la sílice soluble del conglomerante, ni puede determinarse, el método no es aplicable sino a los conglomerados hechos con cemento portland, a base de admitir para éstos la mencionada cifra media de sílice soluble del $20,5 \%$, y el error de la dosificación hallada puede llegar a ser del 18 por ciento.

Esto último es pura teoría basada en unos simples cálculos análogos a los anteriores, pero la realidad es que hay causas "imprevistas" que pueden hacer que los errores sean aún mucho mayores, invalidando el método y sus resultados, como se trata de probar más adelante.

Pero, aparte del caso ideal expuesto, en la realidad se dan casos intermedios en que los áridos de los conglomerados, ni son caliza pura exenta de sílice soluble, ni tampoco canto 
rodado exento de sílice soluble y de óxido cálcico: pueden ser calizas más o menos margosas, rocas más o menos esquistosas o graníticas, etc. En tales casos la solución no es tan inmediata, pues la sílice soluble o el óxido cálcico determinados y encontrados en el conglomerado no provienen exclusivamente del conglomerante. Podría resolverse el problema corrigiendo los valores analíticos hallados para la sílice o la cal en el conglomerante, restando de ellos la parte proporcional correspondiente a los áridos, para lo cual habría que conocer (o determinar por otros medios) la proporción de éstos, y conocer también (o determinar analíticamente) los contenidos de sílice y cal de dichos áridos. Conocer esto es difícil, por no decir imposible, a no ser en casos muy particulares de obras y materiales muy cuidados, controlados y analizados, lo cual se da muy raramente; determinar, es laborioso y exige disponer de muestras representativas de los áridos, lo que también suele ser raro, o extraerlos del propio conglomerado, lo cual hace aún más ardua la resolución del problema. De cualquier modo, los errores a que todo ello da lugar se van sumando y, si en el caso ideal normal el error global podría llegar a ser de $\pm 18 \%$, en los casos reales más frecuentes probablemente es bastante superior.

\section{3 causas de error en casos imprevistos por los métodos}

La Norma A.S.T.M. C 85-54 y, consecuentemente la española NELC 5.01-a precisan con toda claridad, como en otra ocasión y lugar se hizo patente (1), que el método adoptado por ellas, basado en la determinación de la sílice soluble de los conglomerados, atribuible en exclusiva al conglomerante, sólo es aplicable cuando se trata de conglomerados que no contienen áridos o adiciones de materiales silícicos atacables por los ácidos.

Las citadas normas también señalan que el método es aplicable cualquiera que sea el conglomerante empleado en el conglomerado, cuando se conoce el contenido de sílice soluble de aquél, o se dispone de una muestra del mismo para determinarlo. En el caso del cemento portland dicho conocimiento o dicha determinación pueden eludirse adoptando el valor de 20,5 , como contenido porcentual medio de sílice soluble de los cementos portland españoles.

Lo anterior quiere decir implícitamente que, de no disponer del conglomerante ni conocerse su análisis, el método no puede aplicarse a conglomerados hechos con conglomerantes mixtos, entendiéndose por tales los cementos siderúrgicos y los puzolánicos, entre otros, así como tampoco a los hechos con "conglomerantes" que contengan adiciones (activas o inertes) capaces de dar sílice soluble en ácidos. (Para aclarar ideas, en lo sucesivo se designa convencionalmente como "conglomerante" al conjunto de cemento portland y posibles adiciones activas o inertes, y como "cemento" a lo que es única y exclusivamente cemento portland, es decir, clínker y yeso en las debidas proporciones.)

En efecto, si para hallar la dosificación y evitar al máximo los errores se separan lo más perfectamente posible los áridos del hormigón, de lo que pudiera llamarse "mortero enriquecido", admitiendo razonablemente que los áridos separados no contienen nada de "mortero enriquecido", y que éste contiene toda la pasta hidratada del material conglomerante más la parte de áridos que, como consecuencia del proceso mecánico de separación, puediera quedar incluida inseparablemente en él, se tiene que la dosificación $D$ en kilogramos de cemento por metro cúbico de conglomerado vendría dada por: 


$$
\begin{gathered}
D=10 \cdot \bar{Y} \cdot d \\
Y=\frac{\mathrm{MES}}{\mathrm{HTS}} \cdot y \\
y=\frac{\mathrm{SiO}_{{ }^{2} \mathrm{MES}}}{\mathrm{SiO}_{{ }^{2} \mathrm{CAS}}} \cdot 100
\end{gathered}
$$

y en definitiva:

$$
D=10 \cdot \frac{\mathrm{MES}}{\mathrm{HTS}} \cdot \frac{\mathrm{SiO}_{\mathrm{M}_{\mathrm{MES}}}}{\mathrm{SiO}_{2_{\mathrm{CAS}}}} \cdot 100 \cdot d=1.000 \cdot d \cdot \frac{\mathrm{MES}}{\mathrm{HTS}} \cdot \frac{\mathrm{SiO}_{{ }_{\mathrm{MES}}}}{\mathrm{SiO}_{{ }_{\mathrm{CAS}}}}
$$

expresiones en las que $Y$ es el tanto por ciento de "cemento" anhidro seco (CAS) en el hormigón total seco (HTS); MES/HTS la proporción de "mortero enriquecido" seco (MES) en el hormigón total seco (HTS); $\mathrm{SiO}_{2 \mathrm{MES}}$ y $\mathrm{SiO}_{2 \mathrm{CAS}}$ los tantos por ciento de sílice soluble en el mortero enriquecido seco y en el "cemento" anhidro seco, respectivamente, y $d$ la densidad del hormigón seco. Si, por no conocerse $\mathrm{SiO}_{2 \mathrm{CAS}}$, ni poderse determinar al no disponerse del "conglomerante", se le asigna a éste un contenido de sílice soluble del 20,5\%, valor medio admitido por la Norma NELC 5.01-a para los "cementos" españoles, la dosificación calculada será:

$$
D=1.000 \cdot d \cdot \frac{\mathrm{MES}}{\mathrm{HTS}} \cdot \frac{\mathrm{SiO}_{2_{\mathrm{MES}}}}{20,5}=49 \cdot \mathrm{SiO}_{2_{\mathrm{MES}}} \cdot d \cdot \frac{\mathrm{MES}}{\mathrm{HTS}}
$$

Si la sílice soluble del "conglomerante" es mayor que la del "cemento", lo cual sucederá en los casos en que el "conglomerante" sea una mezcla de "cemento" y una adición aportadora de sílice soluble en gran proporción - mayor que la proporción de sílice soluble en el "cemento"-, por ejemplo, una escoria siderúrgica, entonces la dosificación hallada por cálculo será aún mayor que la real. Si, por el contrario, la sílice soluble del "conglomerante" es menor que la del "cemento", lo cual sucederá en los casos en que el "conglomerante" sea una mezcla de "cemento" y una adición aportadora de sílice soluble en una proporción escasa o muy escasa - menor que la proporción de sílice soluble en el "cemento"-, por ejemplo, un crudo, una caliza, una marga, arena de cuarzo, etc., entonces la dosificación de "conglomerante" hallada por cálculo será menor que la real.

Lo anterior puede razonarse cuantitativamente del siguiente modo: Sea C el "conglomerante", $c$ la proporción de "cemento", esto es, de material hidráulico auténticamente cementante contenido en aquél, $e$ la proporción de escoria - material con un contenido porcentual de sílice soluble mayor que el del "cemento" $c-$ y $m$ la proporción de marga calcinada o sin calcinar, crudo, caliza, incocidos, etc. - materiales con un contenido porcentual de sílice soluble menor que el del cemento $c$-, que se encuentran en $C$ en cada caso. Sea también, por fijar ideas, $20,5 \%$ (como en el promedio) el contenido de sílice soluble del "cemento" $c$. Caben los siguientes casos posibles:

1. ${ }^{\circ}$ Si $e=0$ y $m=0, C=c$, y la dosificación del hormigón total seco vendrá dada en función de la relación de los contenidos de sílice soluble en el "mortero enriquecido" seco MES y en el "conglomerante" - o "cemento" - anhidro seco - en este caso $C$ ó $c$ indistintamente-, y, en definitiva, en función del contenido de sílice soluble del primero. Es decir: 


$$
D=\frac{1.000}{20,5} \cdot \mathrm{SiO}_{2_{\mathrm{MES}}} \cdot d \cdot \frac{\mathrm{MES}}{\mathrm{HTS}}=49 \cdot \mathrm{SiO}_{2_{\mathrm{MES}}} \cdot d \cdot \frac{\mathrm{MES}}{\mathrm{HTS}}
$$

En este caso, la dosificación $D$-de "conglomerantes" o de "cemento", indistintamente - hallada, corresponde a la real - como en el caso anterior-, sin más errores que los propios del método analítico y los que tiene en cuenta la norma NELC 5.01-a cuando, en general, el contenido de sílice soluble del conglomerante anhidro difiere de $20,5 \%$, valor aceptado como medio y considerado en este caso.

$2 .^{\circ} \mathrm{Si}$, por ejemplo, $e=15 \%$, y $m=0 \%, C=c+e$, de tal manera que $c=0,85 C$ y $e=0,15 C$. Si, por fijar ideas, se considera que la escoria $e$ contiene $35 \%$ de silice soluble, y el "cemento" $c, 20,5 \%$ - como en el caso anterior-, la sílice soluble del "conglomerante" $C$ será distinta de la del "cemento" $c$. La primera será: $0,85 \times 20,5+0,15 \times 35,0=$ $=22,675$, y la dosificación real de "conglomerante" será:

$$
D=\frac{1.000}{22,675} \cdot \mathrm{SiO}_{2_{\mathrm{MES}}} \cdot d \cdot \frac{\mathrm{MES}}{\mathrm{HTS}}=44,1 \cdot \mathrm{SiO}_{2_{\mathrm{MES}}} \cdot d \cdot \frac{\mathrm{MES}}{\mathrm{HTS}}
$$

Esta dosificación es igual a la anterior [I], pues si bien el primer factor $(44,1)$ está disminuido con respecto al del caso $1 .^{\circ}(49)$, en la relación $22,675 / 20,5=1,106$, el factor $\mathrm{SiO}_{2_{3 I F S}}$ está aumentado con respecto al de dicho caso, precisamente en la misma proporción. Pero, al no conocerse el contenido de sílice soluble del "conglomerante", ni disponer de éste para poderlo determinar, habría que utilizar en la expresión de la dosificación el valor medio de 20,5 según la Norma NELC 5.01-a, con lo cual la dosificación de "conglomerante" calculada sería:

$$
D=49 \cdot \mathrm{SiO}_{2_{\mathrm{MES}}} \cdot d \cdot \frac{\mathrm{MES}}{\mathrm{HTS}}
$$

[III]

distinta y mayor que la anterior [II] y mayor también que la determinada en el caso $10^{\circ}$ [I], puesto que el factor $\mathrm{SiO}_{2 \mathrm{MES}}$ hallado por análisis, en este caso $2 .^{\circ}$ es $22,675 / 20,5=1,106$ veces mayor que en el caso $10^{\circ}$. En cuanto a la dosificación de "cemento", es evidente que la real es el $85 \%$ de la del caso $1 .^{\circ}$, y no se puede determinar experimentalmente, a menos que se conozca la composición del "cemento" y de la escoria, así como sus proporciones respectivas en el "conglomerante", lo cual suele ser aún más difícil que conocer la composición del propio "conglomerante", o disponer de él para determinarla por análisis.

$3 .^{\circ} \mathrm{Si}$, por ejemplo, $e=0 \%$ y $m=15 \%, C=c+m$, de tal manera que $c=0,85 C$ y $m=0,15 C$. Si, por fijar ideas, se considera que la marga, calcinada o no, el incocido, etcétera, $m$, contiene $15 \%$ de sílice soluble, y el cemento $c 20,5 \%$ - como en los casos anteriores-, la sílice soluble del "conglomerante" $C$ será distinta de la sílice soluble del "cemento" c. La primera será: $0,8 \overline{5} \times 20,5+0,15 \times 15,0=19,675$, y la dosificación real de "conglomerante" será :

$$
I=\frac{1.000}{19,675} \cdot \mathrm{SiO}_{2_{\mathrm{MES}}} \cdot d \cdot \frac{\mathrm{MES}}{\mathrm{HTS}}=50,8 \cdot \mathrm{SiO}_{2_{\mathrm{MES}}} \cdot d \cdot \frac{\mathrm{MES}}{\mathrm{HTS}}[\mathrm{IV}]
$$

Esta dosificación será igual a las de los casos anteriores [I] y [II], pues si bien el primer factor $(50,8)$ está aumentado con respecto al del caso $1 .^{\circ}(49)$ en la relación 20,5/19,675= $=1,042$, el factor $\mathrm{SiO}_{2 \mathrm{MLS}}$ está disminuido con respecto al de dicho caso, precisamente en la misma proporción. Pero, al no conocerse el contenido de sílice soluble del "conglomeran- 
te", ni disponer de éste para poderlo determinar, habría que utilizar en la dosificación el valor medio de 20,5 según la Norma NELC 5.01-a, con lo cual la dosificación de "conglomerante" calculada sería:

$$
D=49 \cdot \mathrm{SiO}_{2_{\mathrm{MES}}} \cdot d \cdot \frac{\mathrm{MES}}{\mathrm{HTS}}
$$

distinta y menor que la anterior [IV], y menor también que la determinada en el caso $1 .^{\circ}[\mathrm{I}]$, puesto que el factor $\mathrm{SiO}_{2_{\mathrm{MFS}}}$ hallado por análisis. en este caso $3 .^{\circ}$ es $20,5 / 19,675=$ $=1,042$ veces menor que en el $10^{\circ}$ En cuanto a la dosificación de "cemento", es evidente que la real es el $85 \%$ de la del caso $1 .^{\circ}$, y no se puede determinar experimentalmente, a menos que se conozca la composición del "cemento" y de la marga - en general de la adición--, así como sus proporciones respectivas en el "conglomerante", lo cual suele ser aún más difícil que conocer la composición del propio conglomerante, o disponer de él para determinarla por análisis.

4. ${ }^{\circ}$ Si, por ejemplo, $e=7,5 \%$ y $m=7,5 \%, C=c+e+m$, de tal manera que $c=$ $=0,85 C, e=0,075 C$ y $m=0,075 C$. Si por fijar ideas, se considera que el cemento $c$, la escoria $e$ y la marga, incocido, etc., $m$, tienen los mismos contenidos de sílice soluble que en los casos $2 .^{\circ}$ y $3 .^{\circ}$, es decir, $20,5 \%, 35,0 \%$ y $15,0 \%$, respectivamente, la sílice soluble del “conglomerante" $C$ será distinta de la sílice soluble del "cemento" $c$. La primera será en este caso:

$$
0,85 \times 20,5+0,075 \times 35,0+0,075 \times 15,0=21,175
$$

y la dosificación real de "conglomerante" será:

$$
D=\frac{1.000}{21,175} \cdot \mathrm{SiO}_{2_{\mathrm{MES}}} \cdot d \cdot \frac{\mathrm{MES}}{\mathrm{HTS}}=47,2 \cdot \mathrm{SiO}_{{ }_{\mathrm{MES}}} \cdot d \cdot \frac{\mathrm{MES}}{\mathrm{HTS}}
$$

Esta dosificación será igual a la de los casos anteriores [I], [II] y [IV], pues si bien el primer factor $(47,2)$ está disminuido con respecto al del caso $1 .^{\circ}(49)$, en la relación 21,175 / $/ 20,5==1,033$, el factor $\mathrm{SiO}_{2}$ MES está aumentado con respecto al de dicho caso, precisamente en la misma proporción. Pero, al no conocerse el contenido de sílice soluble del "conglomerante", ni disponer de éste para poderlo determinar, habría que utilizar en la expresión de la dosificación el valor medio de 20,5 según la Norma NELC 5.01-a, con lo cual la dosificación de “conglomerante” calculado será:

$$
D=49 \cdot \mathrm{SiO}_{2_{\mathrm{MES}}} \cdot d \cdot \frac{\mathrm{MES}}{\mathrm{HTS}}
$$

distinta y mayor que la anterior [VI], y mayor también que la determinada en el caso $1 .^{\circ}$, puesto que el factor $\mathrm{SiO}_{2 \mathrm{MEs}}$ hallado por análisis, en este caso $4 .^{\circ}$ es $21,175 / 20,5=1,033$ veces mayor que en el $1 .^{\circ}$. En cuanto a la dosificación de “cemento", es evidente que la real es el $85 \%$ de la del caso $1 .^{\circ}$, y no se puede determinar experimentalmente, a menos que se conozca la composición del cemento, de la escoria y de la marga - en general de las adiciones, o del conjunto formado por todas ellas en la proporción en que intervienen - así como sus proporciones respectivas -o proporción del conjunto- en el "conglomerante", lo cual suele ser aún más difícil que conocer la composición del propio conglomerante, o disponer de él para determinarla por análisis. 
En este último caso $4 .^{\circ}$, según sean las proporciones relativas de escoria $e$ y de marga (o adición en general) $m$, puede ocurrir que, como en el ejemplo expuesto, se halle una dosificación calculada de "conglomerante" mayor que la real, pero puede suceder también que la dosificación calculada sea menor. Es fácil comprobar que tal sucederá siempre que se cumpla la condición $m / e>2,63$. Finalmente, puede ocurrir, para una relación dada entre $e$ y $m$ (y precisamente $m / e=2,63$ ), que el contenido de sílice soluble del "conglomerante" formado por mezcla exclusiva de escoria y marga (o adición), con ausencia absoluta de "cemento", sea igual al contenido de sílice soluble de éste. En efecto:

$$
\begin{gathered}
2,63 \text { partes de } m \cdot \frac{15,0}{100} \text { de } \mathrm{SiO}_{2} \text { en } m+ \\
+1,00 \text { partes de } e \cdot \frac{35,0}{100} \text { de } \mathrm{SiO}_{2} \text { en } e=0,745 \text { partes de } \mathrm{SiO}_{2}
\end{gathered}
$$

que corresponden a $2,63+1,00=3,63$ partes del conjunto $m+e$. A cada parte del mismo

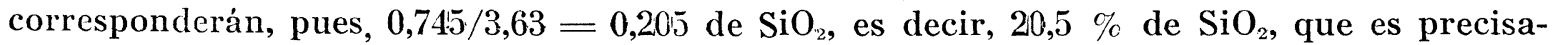
mente el contenido por ciento de sílice soluble del "cemento" $c$. En definitiva, según que $m / e \gtreqless 2,63$, se tendrá una dosificación de “conglomerante” calculada menor, igual o mayor que 1a real, si bien la igualdaà pueae aarse aún en ausencia total de cemento. En efecto, tal sucedería si $c=0, e=27,5 \%$ y $m=72,5 \%$, ya que la sílice soluble del conjunto seria $27,5 \times 35,0 / 100+72,5 \times 15,0 / 100=20,5 \%$ igual a la de "cemento" inexistente. Es decir, que, según lá composición y proporciones de la escoria $e$ y de la marga $m$ (en general de las adiciones) pueden obtenerse "conglomerados" con la dosificación real de "cemento" nula, siendo así que la dosificación determinada y calculada por la Norma NELC 5.01-a puede tener cualquier valor razonable deseado y prefijado. Entre este caso límite por un extremo, y el caso límite del extremo opuesto, que sería el normal de dosificación determinada en un conglornerado hecho con un conglomerante constituido exclusivamente por "cemento", pueden darse todos los casos intermedios.

\section{4 precauciones}

Naturalmente, y por fortuna, que la posibilidad de hacer un conglumerado sin "cemento" es muy remota, y que la de hacerlo "sin que se note" es mucho más remota todavía; podría decirse que esta última es nula. Porque nunca, y menos en casos sospechosos o de controversia, se basa - se puede basar- una dosificación en un solo método que la halle por determinación de un solo componente químico. Y si bien como en el ejemplo del caso $4 .^{\circ}$, expuesto anteriormente, puede darse la circunstancia de "encontrar" una dosificación lógica de "cemento" sin "cemento", basada en el contenido de $\mathrm{SiO}_{2}$, el hallazgo no se sostiene en cuanto se procede a establecer, además, una dosificación basada en el contenido de óxido cálcico, por ejemplo, o de cualquier otro componente "mayor" (5), o simplemente, cuando se verifica un análisis completo de la pastá o del "mortero enriquecido" del conglomerado. $\mathrm{Y}$, por supuesto, cuando se obtienen difractogramas de rayos $\mathrm{X}$ de los mencionados materiales.

Esto sin contar con el caso en que se disponga de una muestra del conglomerante, porque entonces el análisis del mismo deja las cosas en claro. 
Los problemas técnicos, económicos, e incluso jurídicos, a que pueden dar lugar las dosificaciones reales de "cemento" que por un motivo u otro son menores que las nominales en los conglomerados, pueden llegar a ser de gravedad. De problemas técnicos de esta naturaleza se tratará en otra ocasión y lugar, puesto que los demás son de otras competencias.

\section{5 conclusiones}

Los métodos de naturaleza química, establecidos y usualmente empleados para determinar la dosificación de conglomerantes en conglomerados, en general son insuficientes o están sujetos a limitaciones.

Responden aceptablemente a condiciones ideales que prácticamente no se dan nunca, y precisan del conocimiento de determinados datos, el cual no siempre se posee. Alternativamente, exigen disponer de ciertos materiales para determinar analíticamente y directamente dichos datos, lo cual tampoco es factible en la mayor parte de los casos.

Como consecuencia de todo ello, estos métodos llevan implícitas unas ciertas hipótesis "de aproximación" que comportan errores fijos, relativamente fáciles de evaluar en el máximo. Estos errores (previsibles) pueden llegar a ser del orden del 20 por ciento.

Pero, aparte de ello, puede darse - y realmente se dan- otras causas de error en casos imprevistos - y razonablemente imprevisibles - en el planteamiento de dichos métodos. Entre estas causas puede haberlas tales que den lugar a un resultado lógico y aceptable, pero absolutamente irreal, tal como el de encontrar una dosificación normal de cemento en un "conglomerado" que carece de él en absoluto.

En estos casos tales métodos, no sólo son insuficientes, sino que, lo que es peor, pueden inducir a graves errores, con repercusiones no sólo técnicas, sino económicas y de otros órdenes. Entonces y en evitación de dichos errores y de sus consecuencias, es obligado complementar los métodos con otras aportaciones de datos que permitan descartar la posibilidad de aceptación de resultados falsos por irreales. Tal medida de prudencia habrá de extremarse cuando un dictamen basado en el resultado de la dosificación pueda afectar a intereses económicos serios, o determinar una responsabilidad de cualquier tipo.

\section{bibliografia}

1. Calleja, J.: "Determinación de la dosis de cemento en hormigones y morteros endurecidos". I Seminario de Construcción de Carreteras. I.E.T.c.c. Madrid, 1961.

2. Calleja, J.: “Cemento portland: Cálculo e interpretación de datos químicos”. Manuales y Normas del I.E.T.c.c. Madrid, 1960.

3. A.S.T.M. Standards. Método C 85-54 "Cement content of hardened portland cement concrete". 1961.

4. NELC 5.01-a: Normas de Ensayo del Laboratorio Central de Ensayo de Materiales de Construcción. Publicación núm. 42, "Determinación de la dosificación de morteros y hormigones fraguados fabricados con cemento". Madrid, 1947.

5. Ruiz de Gauna, A.: "La determinación de la dosificación de cemento de los morteros y hormigones fraguados, en los casos fuera del ámbito de aplicación de los métodos usuales para hallarla”. Véase este mismo número. 